\title{
Gestión ambiental hotelera en Puerto Vallarta: motivaciones y limitantes
}

\author{
Hotel environmental management in Puerto Vallarta: \\ motivations and barriers
}

Olga Georgina Espinosa Gispert ${ }^{1}$ y Rosa María Chávez-Dagostino ${ }^{2}$

Fecha de recepción: 17 de octubre de 2020

Fecha de aceptación: 11 de enero de 2021

1- Nacionalidad: Mexicana. Grado: Maestría en Ciencias para el Desarrollo, la Sustentabilidad y el Turismo. Adscripción: Universidad de Guadalajara, Campus Centro Universitario de la Costa. (DORCID: https://orcid.org/0000-0003-4477-7404. Correo electrónico: olga.georgina@outlook.com

2- Nacionalidad: Mexicana. Grado: Doctora en Ciencias para el Desarrollo Sustentable. Adscripción: Universidad de Guadalajara, Campus Centro Universitario de la Costa. BORCID: https://orcid.org/0000-0001-9063-2957. Correo electrónico: rosac@cuc.udg.mx 


\section{Resumen}

El deterioro del patrimonio natural en destinos turísticos se ha asociado, entre otros, a las prácticas empresariales. Las acciones enfocadas a la reducción de los impactos ambientales derivados de las actividades de un hotel, constituyen el eje de su gestión ambiental. La certificación ambiental indica la existencia de procesos de gestión, sin embargo, al 2016 menos de la tercera parte de los hoteles de dos a cinco estrellas, tenían una certificación ambiental, por lo que el objetivo de este trabajo fue analizar las motivaciones y limitaciones para desarrollar la gestión ambiental. Mediante entrevistas a profundidad realizadas a actores clave (hoteleros, consultores y gobierno) y procesadas en Atlas ti $v 7$, se encontró que los costos elevados son la limitante más frecuente y la exigencia del cliente es la motivación más nombrada. Estos resultados son consistentes con hallazgos en países como Cuba, España y otras ciudades mexicanas.

Palabras clave: Gestión ambiental, hotelería, obstáculos, motivaciones

\section{Abstract}

The deterioration of the natural heritage in touristic destinations has been associated with business practices. Actions focused on reducing the environmental impact caused by hotels constitute the axis of environmental management. Environmental certification reveals the existence of management processes in this matter; however, in 2016 less than a third of the two-five stars hotels had an environmental certification. The objective of this work was to analyze the drivers and barriers to implement environmental management in hotels. Key actors were interviewed and included hotel staff, consultants, and government agencies. Collected data were processed using Atlas ti $v 7$. Results showed that high costs are the most frequent barrier and the customer's requirements are the most commented driver. The results coincide with findings in Cuba, Spain, and other Mexican cities.

Keywords: Environmental management, hospitality, obstacles, motivations 


\section{Introducción}

E

1 interés de la sociedad por preservar el medio natural se ha incrementado por diversos motivos y los destinos turísticos no están eximidos de esta preocupación, pues dependen de la riqueza natural y cultural que cada uno posee. En el caso de los destinos de sol y playa, si las condiciones ambientales se afectan, mermará la calidad de la experiencia de viaje del turista (Prats, 2011). Esto ocasiona que establecimientos hoteleros adopten medidas que garanticen que se mitigue o evite el impacto ambiental originado por sus actividades, a esto se le conoce como gestión ambiental hotelera (GAH) (Vargas, 2015). La GAH se puede dividir en pública y privada. La GAH pública se refiere a los instrumentos generados por la autoridad para la regulación legal del cuidado ambiental, entre estos instrumentos se encuentra la base jurídica que indica los principios para la protección ambiental en México, la Ley General para el Equilibrio Ecológico y la Protección al Ambiente (LGEEPA), en ella se establecen las acciones que le corresponden a los tres niveles de gobierno: federal, estatal y municipal (Vargas, 2015). Por otro lado, la GAH privada se refiere a las actividades que realiza la empresa (López y Vargas, 2013), ya sean para cumplir con la legislación o por otra motivación relacionada con cuestiones internas o externas de la empresa.

Si bien las empresas de hospedaje están enfocadas a la maximización de ganancias, existen factores que han ocasionado que las compañías re-examinen su ejercicio debido a los nuevos requerimientos internacionales con respecto a la responsabilidad social y la sustentabilidad (Hutchins et al., 2019).

Por otra parte, las buenas prácticas ambientales y las certificaciones son representaciones de acciones relacionadas con el cuidado ambiental, orientadas a respetar la legislación en materia ambiental, según el Programa de Transformación Productiva del Ministerio de Comercio, Industria y Turismo de Colombia (MinCIT, 2014). Ambos instrumentos pueden proveer una fuente de información para examinar la GAH (Reid et al., 2017).

Puerto Vallarta, ciudad y destino turístico en el Estado de Jalisco, México, es considerado como un destino turístico consolidado de índole internacional (Andrade y Chávez-Dagostino, 2007). El inicio de la promoción de Puerto Vallarta como destino turístico fue en la década de los 40, con el fin de evitar la emigración; después se dotó de infraestructura para impulsar el desarrollo de esta región y en las décadas posteriores se realizaron cambios significativos que lo consolidaron, entre éstos, la llegada del turismo masivo asociado a una mayor explotación de los recursos naturales (Scartascini y Rodríguez, 2011). Hoy se reconoce a la actividad turística como su principal vocación en la modalidad de sol y playa, con 3'801,048 turistas nacionales e internacionales hospedados en el 2018 en el municipio de Puerto Vallarta (Secretaría de Turismo Jalisco, 2019). Esta vocación ha traído beneficios como la ampliación y diversificación de empleos, equipamiento y formación de capacidades humanas. Sin embargo, también trajo consigo externalidades como "la afectación al medio natural, la normalización de prácticas donde los intereses privados se imponen sobre los bienes comunes y la adopción de un sistema turístico donde la cantidad es más importante que la calidad de la experiencia ..." (Baños Francia et al., 2020, p. 54).

Según la Secretaría de Turismo en México, en el municipio de Puerto Vallarta existían 131 establecimientos de hospedaje, pertenecientes a categorías de dos, tres, cuatro y cinco estrellas donde menos de la tercera parte contaban con alguna certificación ambiental (Secretaría de Turismo Jalisco, 2016). Este 
hecho permite cuestionar el porqué de la existencia de hoteles que practican la gestión ambiental y otros establecimientos que no lo hacen, pues entender los obstáculos y las motivaciones para realizar acciones de protección ambiental en los establecimientos, puede permitir generar estrategias para que más hoteles se integren al cuidado ambiental o, en su caso, visualizar las complicaciones para establecer tácticas y minimizar estas limitaciones. Comprender lo que motiva y limita a un hotel para implementar buenas prácticas ambientales y obtener certificaciones tiene implicaciones significativas para los dueños, administradores y empleados de las empresas, así como también para consultores y para los responsables de formular política pública, que al final está relacionada con la economía local de los países donde operan (Toledo y Gopar, 2012). Un actor puede afectar o ser afectado por los logros de la empresa, dentro de la empresa se encuentran: empleados, gerentes, propietarios, accionistas. Fuera de la empresa están: el gobierno, la sociedad, competidores, asociaciones no gubernamentales y clientes (Toledo y Gopar, 2012).

Es por lo anterior que el objetivo de este estudio fue analizar las motivaciones y limitaciones para realizar gestión ambiental en hoteles de Puerto Vallarta, desde la perspectiva de diferentes actores. Para ello se realiza revisión de la literatura de la gestión ambiental hotelera (GAH) y una indagación de lo que algunos autores han encontrado sobre lo que motiva y obstaculiza a un hotel, para realizar gestión ambiental en sus establecimientos.

\section{Revisión de la literatura}

A nivel internacional se han establecido iniciativas y programas en relación con la protección ambiental, esto ha permeado en la industria, incluyendo al sector hotelero y restaurantes principalmente (Kirk, 1995). Esto ha sido incentivado fuertemente a partir de la instalación de los Objetivos de Desarrollo del Milenio que promovieron entre otros, la protección de nuestro entorno común (Organización de las Naciones Unidas [ONU], 2000) y posteriormente los Objetivos de Desarrollo Sostenible que refrendan estos objetivos con mayor énfasis. Por otra parte, en la Agenda 2030 para el Desarrollo Sostenible se establecen metas de carácter integrado e indivisible relacionadas con la gestión del agua, la energía, el cambio climático y la conservación de la biodiversidad entre otros (ONU, 2019).

Paralelamente se han detonado prácticas ambientales relacionadas con la competitividad de los destinos turísticos, donde el concepto de sustentabilidad ha sido utilizado para generar estrategias en diversos sectores (Higgins-Desbiolles, 2018), especialmente en el tema ambiental.

Casi todos los países disponen de una legislación en materia ambiental, con derechos y obligaciones ciudadanas, funciones de Estado y organismos públicos en materia ambiental (Rodríguez-Becerra y Espinoza, 2002). Para el caso mexicano, en 1971 se creó la Ley Federal para Prevenir y Controlar la Contaminación Ambiental, siendo este el primer ordenamiento jurídico mexicano de naturaleza ambiental (Pérez, 2010). El contexto internacional influyó en la legislación mexicana y se decreta una nueva ley en 1988: Ley General del Equilibrio Ecológico y la Protección al Ambiente (LGEEPA), siendo ésta la base para la regulación ambiental en México, de la que se desprenden una serie de leyes, reglamentos y normas. Esta regulación promueve la gestión ambiental que se ve reflejada por la conformación de políticas ambientales en los negocios (Cramer, 1998), una forma incipiente de vinculación entre el desarrollo y el ambiente. 
Hace poco más de dos décadas aparecieron los esquemas de certificación para la industria y el turismo. Varios de estos esquemas fueron creados alrededor del mundo por el crecimiento del consumo ambiental, pues la idea de adquirir productos o servicios que son ambientalmente responsables se ha instalado en la psique del consumidor (Dunk et al., 2016). Es así que los servicios de hospedaje adquieren interés por el cuidado del ambiente en función de los imperativos del corporativo, cumplimiento de regulación ambiental, demandas del cliente y presiones de directivos, entre otras (Chan y Hsu, 2016).

En 1987 ya existía el concepto de gestión ambiental, entendido como "elemento teórico y técnico que integra las acciones normativas, administrativas y operativas" (Vargas et al., 2011, p.186). Aunque hay variaciones en la definición, la idea ha seguido siendo la misma. En los años 90 se decía que la gestión ambiental involucra tanto al nivel administrativo como operacional, donde las actividades de la organización tienen como meta reducir los impactos ambientales ocasionados por la operación de las compañías (Cramer, 1998). Se puede definir entonces a la gestión ambiental hotelera como el proceso de realizar acciones enfocadas a las actividades que pueden ocasionar impactos ambientales; puede ser pública, la cual se implementa mediante instrumentos tales como: políticas, leyes e inspecciones (López y Vargas, 2013) o privada, llevada a cabo mediante acciones voluntarias dentro de las empresas.

Un establecimiento hotelero utiliza recursos diversos como energía, agua, productos no durables, genera residuos sólidos y aguas residuales (Cingoski y Petrevska, 2018).

Por otro lado, el patrimonio natural en los destinos turísticos es parte importante de su atractivo, no obstante, la empresa hotelera ha sido objeto de críticas en cuanto a su participación en el deterioro del recurso natural que lo rodea. Sin embargo, también han sido consideradas como un sector dispuesto y preocupado por la conservación del ambiente natural, integrando iniciativas voluntarias de gestión ambiental (Vargas et al., 2011).

Además de implementarse acciones para la prevención y control de los procesos que generan impactos ambientales, la gestión ambiental también incluye actividades de organización y comunicación, tanto con los grupos internos como los externos al hotel (Vargas, 2015), considerando el factor de ahorro económico en los proyectos que se implementen y en ocasiones el ámbito de responsabilidad social (Vargas et al., 2011). Para ello, las empresas hoteleras se apoyan de diferentes procesos como la adopción de sistemas de gestión ambiental, obtención de certificaciones o buenas prácticas ambientales, entre otros (Ortiz et al., 2016).

Es así que los enfoques de gestión ambiental no son homogéneos porque dependen de numerosos factores determinantes, como los recursos organizativos, los valores de gestión o las condiciones del mercado (Bagur-Femenias et al., 2016). Los instrumentos de gestión ambiental empleados en el sector hotelero son diversos, los más utilizados son las buenas prácticas ambientales y las certificaciones (Navarrete y Zanfardini, 2014).

A lo largo del tiempo se han propuesto indicadores de gestión ambiental incluidos en diferentes dimensiones (Cuadro 1), no son homogéneas en cuanto a escalas y enfoques, varían de acuerdo a los autores. Aunque las dimensiones de gestión ambiental pueden diferir nominalmente, los indicadores derivados frecuentemente son similares, incluyendo a los utilizados en establecimientos de hospedaje. Es común también que los trabajos relacionados no mencionen la palabra dimensión o enfoque y solamente utilicen una batería de preguntas e indicadores como el trabajo de Wan et al. (2017). El trabajo de estos autores incluyó indicadores para evaluar la conciencia ambiental de los administradores de diversas áreas 
en los hoteles de Macau, la implementación de buenas prácticas ambientales y las estrategias orientadas a la educación ambiental de sus clientes.

\section{Cuadro 1}

Algunas dimensiones de la gestión ambiental utilizadas en empresas hoteleras

\begin{tabular}{|c|c|c|}
\hline Autores & Dimensiones propuestas & Explicación \\
\hline Mihalic (2000) & $\begin{array}{l}\text { Códigos de conducta ambiental } \\
\text { Prácticas ambientales } \\
\text { Esquemas de certificación }\end{array}$ & $\begin{array}{c}\text { Códigos de conducta: consultar a los actores clave, } \\
\text { capacitación de personal, apoyo a la comunidad local y } \\
\text { reducción del consumo de recursos. } \\
\text { Prácticas ambientales: Actividades y programas inde- } \\
\text { pendientes, es decir sin necesidad de un reconocimiento } \\
\text { externo. } \\
\text { Esquemas de certificación: Programas de acreditación } \\
\text { por un externo. }\end{array}$ \\
\hline \multirow{2}{*}{$\begin{array}{l}\text { Álvarez, Burgos } \\
\text { Jiménez y Céspedes } \\
\text { Lorente (2001) }\end{array}$} & Gestión ambiental tácita & $\begin{array}{l}\text { Gestión ambiental tácita: Cuando la motivación predo- } \\
\text { minante no es la protección ambiental. }\end{array}$ \\
\hline & Gestión ambiental expresa & $\begin{array}{c}\text { Gestión ambiental expresa: Cuando la motivación pre- } \\
\text { dominante para realizar gestión ambiental es por cuidar } \\
\text { el medio ambiente. }\end{array}$ \\
\hline $\begin{array}{l}\text { Céspedes y De Bur- } \\
\text { gos (2004) }\end{array}$ & $\begin{array}{l}\text { Gestión Ambiental Avanzada } \\
\text { (GAA) } \\
\text { Gestión Ambiental Mínima } \\
\text { (GAM) }\end{array}$ & $\begin{array}{l}\text { GAA: Hoteles que proponen estrategias de protección } \\
\text { ambiental las aprovechan } \\
\text { como una herramienta de competitividad. } \\
\text { GAM: Hoteles que establecen acciones fáciles de ejecu- } \\
\text { tar generalmente asociadas a costos y competitividad. }\end{array}$ \\
\hline $\begin{array}{l}\text { López y Vargas } \\
\text { (2013) }\end{array}$ & $\begin{array}{l}\text { Acción } \\
\text { Comunicación } \\
\text { Disposición } \\
\text { Monitoreo }\end{array}$ & $\begin{array}{l}\text { Acción: Estándares, auditorías ambientales y capacita- } \\
\text { ción ambiental. } \\
\text { Comunicación: La organización dentro del hotel para } \\
\text { transmitir información a huéspedes y empleados. } \\
\text { Disposición: Vínculos con la autoridad para el cumpli- } \\
\text { miento de la legislación, conocimiento de los beneficios } \\
\text { e intención de realizar acciones. } \\
\text { Monitoreo: monitoreos de consumo de residuos, agua y } \\
\text { energía. }\end{array}$ \\
\hline Vargas (2015) & $\begin{array}{l}\text { Gestión ambiental empresarial } \\
\text { Gestión ambiental pública }\end{array}$ & $\begin{array}{c}\text { Gestión ambiental empresarial: las acciones realizadas } \\
\text { por la empresa privada. } \\
\text { Gestión ambiental pública: Regulación ambiental } \\
\text { mexicana. }\end{array}$ \\
\hline $\begin{array}{l}\text { Bagur-Femenias, } \\
\text { Celma y Patau } \\
\text { (2016) }\end{array}$ & $\begin{array}{l}\text { Prácticas ambientales } \\
\text { Desempeño operacional } \\
\text { Competitividad }\end{array}$ & $\begin{array}{c}\text { Prácticas ambientales: Implementación de acciones } \\
\text { para la protección ambiental. } \\
\text { Desempeño operacional: Las acciones que adoptan las } \\
\text { compañías para ahorro económico, salud y seguridad } \\
\text { del personal. } \\
\text { Competitividad: Mejora de la imagen desde la perspec- } \\
\text { tiva interna y externa. }\end{array}$ \\
\hline
\end{tabular}

Fuente: Elaboración propia basada en los trabajos citados. 
La auditoría de las buenas prácticas ambientales es una evaluación que puede concluir con la obtención de una certificación (Vargas y Olivares, 2012).

Las certificaciones pueden ser internacionales, nacionales o estatales, las obtenidas por hoteles de Puerto Vallarta son usualmente internacionales como Green Globe, Earth Check, Rainforest Alliance y Green Key. Las certificaciones como norma ISO 14001 y reglamentos EMAS (Eco-Management and Audit Scheme) permiten a las compañías hoteleras identificar y mitigar el impacto ambiental de sus servicios (Valenzuela, 2017). La certificación nacional es denominada Calidad Ambiental Turística, otorgada por la Procuraduría Federal de Protección al Ambiente y el reconocimiento estatal en Jalisco es el Programa de Cumplimiento Ambiental Voluntario de la Secretaría de Medio Ambiente y Desarrollo Territorial.

Ahora bien, se pueden llevar a cabo buenas prácticas ambientales sin necesidad de una certificación. Las buenas prácticas ambientales tienen la finalidad de mejorar el ambiente en el entorno de la empresa, son un paso para sensibilizar a los integrantes del hotel en materia ambiental (Andrés y Torre, 2010). Tanto la certificación como la práctica ambiental buscan mitigar los impactos, sin embargo, la primera es un reconocimiento otorgado por un tercero y la segunda son acciones que no necesariamente llegan a obtener un reconocimiento externo, es decir, fuera de la misma empresa; así pues, cada establecimiento elige si realizar gestión ambiental o si, por el contrario, no la considera un elemento importante.

\subsection{Motivaciones y limitaciones para la gestión ambiental}

Existen diversas razones reconocidas para que un hotel establezca un sistema de gestión ambiental, pueden ser motivos internos o externos (Carasuk et al., 2013). Entre estas razones se encuentran el ahorro económico (Aznar et al., 2016), la reparación u optimización de la imagen (Ortiz et al, 2016), la satisfacción de las expectativas del consumidor (Emre et al., 2015), el cumplimiento de la legislación (Vargas et al., 2011), mantener una relación directa con la autoridad (Chiappetta et al., 2016), el establecimiento de vínculos con el gobierno local al implementar prácticas de ahorro de agua, energía o reducción de residuos (López y Vargas, 2013), a solicitud por parte de directivos o del corporativo (Navarrete y Zanfardini, 2014), o por motivos éticos (Peiró-Signes et al., 2014).

Generalmente, se le otorga más importancia al ahorro en costos e incremento en ganancias, que a la voluntad del huésped (Mihalič, 2000). Sin embargo, también le importa a la empresa tener la capacidad para generar un valor agregado en su establecimiento, por lo cual implementan acciones para aumentar su valor social y ambiental, reteniendo así a clientes leales (Peiró-Signes et al, 2014). Entonces, el cliente es un factor para la adquisición de certificaciones y buenas prácticas ambientales, pero no siempre es el factor determinante (Peiró-Signes et al., 2014). Según López y Vargas (2013), los directivos hoteleros perciben que el cumplimiento de la legislación y la presión por parte de las autoridades en materia ambiental, son detonantes para la realización de la GAH.

Cuando un hotel ya se ha decidido por realizar prácticas para el cuidado del medio ambiente, los empleados serán los que implementen las actividades, por lo cual su participación es crucial para el logro de las iniciativas (Rubin et al., 2012). Los dueños de hoteles aprecian las prácticas para el cuidado ambiental, incluyendo el bajar los costos operacionales, mejorar la imagen de la compañía y reducir sus consumos de luz y agua, mitigando su impacto ambiental (Wan, et al., 2017). 
En cuanto a las limitaciones se refiere, estudios anteriores han encontrado que los costos de gestión ambiental son una barrera significativa para implementar medidas de GAH, particularmente en el sector servicios. Así mismo, los estudios previos también han catalogado como limitante a los beneficios inciertos de la implementación de acciones de protección ambiental; la falta de conocimiento del personal; la inversión de tiempo y la ausencia de una recompensa tangible para los empleados se ve a veces como un factor desmotivante (Ervin et al., 2012). Asimismo, los huéspedes no siempre consideran reservar en un hotel con buenas prácticas ambientales y la falta de inspección por la autoridad también es una barrera, por lo que no se convierte en motivación para realizar gestión ambiental hotelera (Wan et al., 2017).

Algunos hoteles, sobre todo los pequeños, pueden tener un compromiso con el ambiente y no necesariamente obtener una certificación, por los costos que esto implica (Sánchez-Medina et al., 2015).

La acción de una empresa referente a los problemas ambientales depende de varios factores: la falta de disponibilidad de capital para realizar inversiones de mejora ambiental, ausencia de conocimiento técnico, el costo y el tiempo para el cambio de actitudes del personal, esta resistencia se considera como una barrera, pues la implementación de nuevas actividades puede generar molestia entre los empleados (Navarrete y Zanfardini, 2014). La falta de coordinación entre las secretarías (Vargas, 2015), se considera también una limitación, pues cuando no existen acuerdos entre las autoridades, ya sea para inspeccionar o para incentivar a la empresas a cumplir con la legislación, existe una ruptura que dificulta la adopción de gestión ambiental en los establecimientos de hospedaje. Por otro lado, a veces no existe interés de adoptar buenas prácticas ambientales cuando la inspección por parte de la autoridad está ausente (Fleig et al., 2015).

Existe también cierto riesgo durante la implementación de las acciones, es decir, el proceso puede llegar a abandonarse al no ser instantáneos los resultados; también estos factores pueden representar costos en materiales, equipo y recurso humano (Ervin et al., 2012), autores también han reportado dificultades relacionadas con el seguimiento de los estándares en las certificaciones por motivo de la complejidad o la falta de incentivos. El cambio de hábito de los empleados, la falta de comunicación y el liderazgo realizado inadecuadamente por parte de la alta dirección, son otras barreras mencionadas por autores (Chan, 2008). También para López y Vargas (2013), quiénes realizaron un estudio en Cuba, la ausencia de monitoreo dentro de la gestión ambiental y la falta de comunicación, ocasionan que no se difunda entre clientes y proveedores acerca de las actividades con las que el establecimiento contribuye para la protección ambiental; al no ser externada la información, los clientes y proveedores carecen de dichos datos.

Un estudio realizado por Valenzuela (2017) en establecimientos hoteleros españoles, menciona que las cadenas hoteleras han demostrado un mayor interés para la implantación de buenas prácticas ambientales y certificaciones debido a su solvencia económica y el interés por cuidar su imagen.

La investigación de este artículo representa la búsqueda de las razones para realizar o no, gestión ambiental hotelera en Puerto Vallarta.

\section{Metodología}

Es un estudio de corte cualitativo y se utilizaron como instrumentos de investigación la recopilación documental y la entrevista semiestructurada de acuerdo a lo propuesto por Wan et al. (2017). La entre- 
vista "cara a cara" se hizo por invitación a través de la Dirección de Hoteles y Moteles de Puerto Vallarta y el muestro fue de tipo no aleatorio por conveniencia (Otzen y Manterola, 2017).

Se identificaron como actores clave a los organismos gubernamentales relacionados con la aplicación de la legislación ambiental, a los consultores en materia ambiental por su conocimiento en el tema y al personal hotelero (directores de área, gerentes o supervisores) por ejercer la actividad relacionada (Almada, 2008). El personal hotelero entrevistado pertenecía a un alojamiento con categoría: 2, 3, 4 o 5 estrellas (Cuadro 2).

\section{Cuadro 2 \\ Perfil de actores clave entrevistados}

\begin{tabular}{|c|c|}
\hline Actor clave & \multicolumn{1}{|c|}{ Perfil } \\
\hline Consultores & $\begin{array}{c}\text { Experiencia profesional en asesoría a empresas hotele- } \\
\text { ras en materia ambiental }\end{array}$ \\
\hline Organismos gubernamentales & $\begin{array}{c}\text { Autoridades de instancias gubernamentales ambienta- } \\
\text { les: municipal, estatal y federal }\end{array}$ \\
\hline Personal de establecimientos hoteleros & $\begin{array}{c}\text { Responsables de decisiones directivas en la empre- } \\
\text { sa: gerencia general, gerencia de recursos humanos, } \\
\text { gerencia de calidad y gerencia de recepción }\end{array}$ \\
\hline
\end{tabular}

Fuente: Elaboración propia

Según la Secretaría de Turismo Jalisco (2016), 131 establecimientos de hospedaje registrados en Puerto Vallarta se manejan con alguna categoría entre dos y cinco estrellas. Para seleccionar la muestra de establecimientos de hospedaje, se procedió a considerar a todas las categorías, entrevistando a dos hoteles mínimo por categoría. Dentro de esta muestra seleccionada se entrevistaron a establecimientos que contaban con una certificación ambiental y a hoteles que no la tenían (Cuadro 3).

\section{Cuadro 3}

Hoteles entrevistados

\begin{tabular}{|c|c|c|c|}
\hline Categoría de hotel & Número de hoteles entrevistados & Certificados & No certificados \\
\hline 5 estrellas & 2 & 1 & 1 \\
\hline 4 estrellas & 4 & 2 & 2 \\
\hline 3 estrellas & 2 & 1 & 1 \\
\hline 2 estrellas & 2 & 0 & 2 \\
\hline
\end{tabular}

La duración de cada entrevista osciló entre cuarenta minutos y una hora, no siendo necesaria una segunda entrevista para ninguno de los entrevistados. La temporalidad de la recolección de información fue de cinco meses (de noviembre 2017 a abril 2018), debido a que las personas entrevistadas proponían horario y fecha. 
En este caso se consideraron un total de 18 actores clave que aceptaron participar, distribuidos de la siguiente manera: cuatro de organismos gubernamentales, cuatro prestadores de servicios y diez correspondientes al personal hotelero.

En la aplicación de las entrevistas previa cita, se procedió de la siguiente manera: se explicó al entrevistado la dinámica, se solicitó permiso para grabar audio, se mantuvo una actitud receptiva y se le permitió al entrevistado hablar de manera libre; todo ello con preguntas flexibles previamente estructuradas (Díaz-Bravo et al., 2013). Las entrevistas incluyeron temas diferenciados para cada sector (Cuadro 4).

Cuadro 4

Temas abordados en las entrevistas semiestructuradas por tipo de actor

\begin{tabular}{|c|c|c|}
\hline Prestadores de servicio & Organismos gubernamentales & Personal hotelero \\
$\begin{array}{c}\text { ¿Por qué los hoteles realizan } \\
\text { prácticas para el cuidado del medio } \\
\text { ambiente? }\end{array}$ & $\begin{array}{c}\text { ¿En qué interviene este organis- } \\
\text { mo en la gestión ambiental de los } \\
\text { hoteles? }\end{array}$ & $\begin{array}{c}\text { ¿Cuáles prácticas ambientales reali- } \\
\text { zan en su establecimiento? }\end{array}$ \\
$\begin{array}{c}\text { ¿Qué resistencias u obstáculos exis- } \\
\text { ten para que los hoteles realicen } \\
\text { prácticas de gestión ambiental? }\end{array}$ & $\begin{array}{c}\text { ¿Por qué es importante la gestión } \\
\text { ambiental en Puerto Vallarta, sobre } \\
\text { todo en los hoteles? }\end{array}$ & $\begin{array}{c}\text { ¿Por qué realizan acciones para el } \\
\text { cuidado del medio ambiente? }\end{array}$ \\
\hline $\begin{array}{c}\text { ¿El interés de los hoteles por llevar } \\
\text { a cabo estas prácticas es mayor, } \\
\text { menor o estable? }\end{array}$ & $\begin{array}{c}\text { ¿Cuáles son los obstáculos para } \\
\text { que los hoteles realicen gestión } \\
\text { ambiental? }\end{array}$ & $\begin{array}{c}\text { ¿Han existido complicaciones para } \\
\text { realizar estas prácticas? }\end{array}$ \\
\hline $\begin{array}{c}\text { ¿Cuáles son las prácticas más co- } \\
\text { munes que realiza un hotel? }\end{array}$ & $\begin{array}{c}\text { ¿Cuáles son los beneficios para el } \\
\text { hotel al realizar prácticas de protec- } \\
\text { ción ambiental? }\end{array}$ & $\begin{array}{c}\text { Recuerda si, ¿alguna vez un hués- } \\
\text { ped ha estado interesado en las } \\
\text { prácticas que realiza el hotel? }\end{array}$ \\
\hline
\end{tabular}

Fuente: Elaboración propia basada en preguntas realizadas por autores en investigaciones similares (Wan, et al., 2017; Chan et al., 2018; Walker et al., 2008).

La información de las entrevistas semiestructuradas se categorizó y codificó (Wan et al., 2017), analizándose con la herramienta Atlas.ti versión 7. El análisis de las entrevistas semi estructuradas se inició con la identificación de uno o más pasajes dentro del texto, relacionado con limitaciones y motivaciones, luego se asignaron códigos que describen las frases identificadas dentro del texto. El producto fue un informe de limitaciones y motivaciones, previamente seleccionadas. A partir de este informe se determinaron los factores que los actores clave perciben como barreras para realizar la gestión y los elementos que representan una motivación para efectuar acciones de gestión ambiental en los establecimientos de hospedaje. Aunque el objetivo no era hacer comparaciones entre las diferentes categorías de hoteles por el tipo de muestra, se hacen algunas consideraciones narrativas al respecto. 


\section{Resultados y discusión}

El análisis de las limitaciones reveló la existencia de tres externas: falta de interés del cliente, falta de lineamientos y ausencia de inspección. Igualmente se mencionaron tres limitaciones internas: costos elevados, falta de conocimiento técnico y falta de participación directiva. En cuanto a las motivaciones se refiere, los actores clave perciben como motivaciones externas a la relación directa con la autoridad, la imagen, el cumplimiento de la legislación y la competitividad. Asimismo, se reconocieron como motivación interna para realizar GAH cuatro factores: ahorro económico, cumplimento de estándares, participación directiva y motivos éticos. Para llegar a ello la opinión de los actores clave se catalogó por conceptos, mismos que se clasificaron como limitaciones internas ( $\mathrm{Li})$, limitaciones externas (Le), motivaciones internas ( $\mathrm{Mi}$ ) y motivaciones externas (Me) (Cuadro 5).

Cuadro 5

Limitaciones y motivaciones de gestión ambiental por actor clave, en hoteles de Puerto Vallarta

\begin{tabular}{|c|c|c|c|c|}
\hline Concepto & Frases mencionadas en las entrevistas & Estrellas & Actor & Categoría \\
\hline \multirow{13}{*}{ Cliente } & "Poca gente tiene esta conciencia" & 2 & \multirow{5}{*}{ Hotelero } & \multirow{5}{*}{ Le } \\
\hline & "Es difícil que el cliente se interese" & 5 & & \\
\hline & "A veces al huésped no le interesa" & 2 & & \\
\hline & "El huésped no participa" & 4 & & \\
\hline & "Si existe cultura, pero aún falta" & 5 & & \\
\hline & "Existe la cultura por parte de extranjeros" & 3 & \multirow{5}{*}{ Hotelero } & \multirow{8}{*}{ Me } \\
\hline & "Si hay huéspedes interesados" & 3 & & \\
\hline & "Ofrecerle al cliente lo que busca" & 4 & & \\
\hline & "Comentan en Trip advisor sobre esto" & 4 & & \\
\hline & $\begin{array}{c}\text { "Buscan lugar certificado" y "el cliente lo } \\
\text { exige" }\end{array}$ & 5 & & \\
\hline & "Lo demanda la gente" y "el turista lo pide" & - & \multirow[t]{2}{*}{ Gobierno } & \\
\hline & “Preguntan sobre el tema" & - & & \\
\hline & "El turista exige el cuidado al ambiente" & - & Consultor & \\
\hline
\end{tabular}




\begin{tabular}{|c|c|c|c|c|}
\hline Concepto & Frases mencionadas en las entrevistas & Estrellas & Actor & Categoría \\
\hline \multirow{5}{*}{ Costos elevados } & "Los costos deben cuidarse" & 3 & Hotelero & \multirow{5}{*}{ Li } \\
\hline & "Evaluar la existencia de un beneficio" & 3 & Hotelero & \\
\hline & "Alto costo de inversión" & 5 & Hotelero & \\
\hline & "Implica una fuerte cantidad de dinero" & - & Gobierno & \\
\hline & “La implementación es costosa" & - & Consultor & \\
\hline \multirow{5}{*}{ Ahorro económico } & "Minimizar el costo de la electricidad" & 2 & & \multirow{5}{*}{ Mi } \\
\hline & "Que económicamente convenga" & 3 & & \\
\hline & "Lograr economizar" y "generar ahorros" & 3 y 4 & Hotelero & \\
\hline & "Entre más se ahorre es mejor" & 4 & & \\
\hline & "Evitar multas" & - & Gobierno & \\
\hline \multirow{4}{*}{ Imagen } & "Cuestión de mercadotecnia" & 3 & Hotelero & \multirow{4}{*}{ Me } \\
\hline & "Impactar al turista mediáticamente" & - & \multirow{3}{*}{ Gobierno } & \\
\hline & “Les sirve para marketing” & - & & \\
\hline & "Le conviene al hotel por imagen" & - & & \\
\hline \multirow{5}{*}{ Participación directiva } & "Son iniciativas del director general" & 5 & \multirow{3}{*}{ Hotelero } & \multirow{3}{*}{ Mi } \\
\hline & "Los directivos apoyan los proyectos" & 5 & & \\
\hline & "Los directivos están convencidos" & 4 & & \\
\hline & "Falta interés por parte de los dueños" & 4 & Hotelero & \\
\hline & “Esencial: dirección no apoya” & - & Consultor & LI \\
\hline \multirow{3}{*}{$\begin{array}{l}\text { Requisito: legal o cor- } \\
\text { porativo }\end{array}$} & "Para cumplir con la legislación” & 4 & Hotelero & \multirow{2}{*}{ Me } \\
\hline & “Por el marco legal” & - & Consultor & \\
\hline & "Son iniciativas del corporativo" & 4 y 5 & Hotelero & Mi \\
\hline \multirow{2}{*}{ Relación con autoridad } & “Trabajar junto con la autoridad” & 5 & Hotelero & \multirow{2}{*}{ Me } \\
\hline & "Contacto más directo" & - & Gobierno & \\
\hline \multirow{2}{*}{ Falta noción técnica } & "Falta de información" & - & Consultor & \multirow{2}{*}{ Li } \\
\hline & "No forma parte de la operación" & - & Gobierno & \\
\hline Competitividad & “Ventaja competitiva” & - & Consultor & Me \\
\hline Motivos éticos & "Ser respetuosos con el ambiente" & 5 & Hotelero & Mi \\
\hline Faltan lineamientos & "Lugares no registrados como hotel” & - & Gobierno & Le \\
\hline Inspección & “Falta de inspección” & - & Consultor & Le \\
\hline
\end{tabular}

Fuente: Elaboración propia a partir de la información obtenida del presente estudio 
Con respecto a la información obtenida, puede establecerse que el cliente representa tanto una limitación como motivación; si se compara la visión de los actores clave y la frecuencia con que se mencionan estos, la presión del cliente representa más una motivación que una limitación; aunque no siempre está presente esta presión externa hacia el hotel como para realizar GAH por este móvil, concordando esto con un estudio realizado por Bruns-Smith et al. (2015), donde también la satisfacción del cliente en cuanto a las buenas prácticas ambientales se considera débil. Además, los establecimientos que mencionan al cliente meramente como una limitación por su falta de interés son los establecimientos de categoría dos estrellas.

La motivación más comentada por los actores clave entrevistados, después del cliente, es el ahorro económico. Según Aznar et al. (2016), se requiere una existencia consistente de administración y poder adquisitivo (costos operacionales) para la obtención de una certificación ambiental, mencionan también que a corto plazo pueden no observarse en los ingresos del establecimiento, pero sí a largo plazo. Todos los establecimientos hoteleros entrevistados, sean de dos, tres, cuatro o cinco estrellas, concuerdan que el ahorro económico es una razón de peso para realizar gestión ambiental hotelera. Por otro lado, la imagen se visualiza como una motivación externa para realizar GAH, según los actores clave entrevistados. Esto concuerda con Vargas et al. (2011), pues mencionan que la imagen externa es un factor motivante para llevar a cabo gestión ambiental hotelera para los establecimientos de hospedaje de Cancún.

La participación directiva es mencionada como una limitación cuando está ausente y como una motivación cuando desde los altos rangos se le otorga importancia a la protección ambiental. Los hoteles de cuatro y cinco estrellas entrevistados son los que mencionan a la participación directiva como esencial para realizar prácticas de protección ambiental. En cuanto a la parte legal se refiere, el cumplimiento de las obligaciones ambientales es una motivación para la realización de GAH, según actores clave, los autores Bagur-Femenias et al. (2016) comentan que, para que se cumpla con la legislación ambiental, es necesario contar con inspección; concordando con la visión de un actor clave del sector de consultoría, la falta de esta se vuelve una limitación; los hoteles de categoría cuatro y cinco estrellas mencionan al cumplimiento legal como una motivación para realizar gestión ambiental hotelera. Asimismo, la falta de conocimiento técnico se visualiza como una limitación interna para realizar GAH. Cabe agregar que el hecho de que un hotel pertenezca a una cadena hotelera le da fortaleza para realizar prácticas para el cuidado ambiental, pues como mencionan Álvarez et al. (2001) en un análisis de gestión ambiental que realizaron a hoteles de España, el rendimiento financiero se relaciona con el tamaño del hotel (cadenas hoteleras). Dos actores clave mencionan que se lleva GAH como un requisito por parte del corporativo, ambos pertenecen a categoría superior a tres estrellas, es decir, son de cuatro y cinco estrellas.

Es importante señalar que uno de los actores clave (consultor), estableció que la competitividad es un factor para aplicar acciones de cuidado ambiental y así mismo, solamente se menciona una vez que las actividades se realizan por motivos éticos, refiriéndose a que una de las razones para realizar buenas prácticas ambientales es por convicción, por conciencia. Finalmente, se percibe como limitación externa la falta de lineamientos, refiriéndose a aquellos cuartos que se rentan mediante plataformas.

Con respecto a los trabajos revisados, se encontraron coincidencias en las limitantes como la falta de regulación legal en materia ambiental y la dificultad de encontrar un balance con la expectativa del cliente y las prácticas ambientales (Wan et al., 2017). Para el caso de hoteles en Barcelona, según Aznar et al. (2016) existe un crecimiento de conciencia ambiental en los huéspedes, sin embargo, la presión para que se adopte 
gestión ambiental en un hotel por esta razón, es pequeña; concordando con lo que se encontró en el presente estudio. Asimismo, en establecimientos de hospedaje de Barcelona, los hoteles necesitan un poder adquisitivo para contar con una certificación ambiental, razón que concuerda con la visión de actores clave entrevistados, pues comentan que las iniciativas ambientales requieren una inversión elevada.

Por otra parte, para establecimientos de hospedaje de Varadero, Cuba, la principal motivación representa el cumplimiento legal ambiental, mientras que para este estudio es importante para los hoteles con categoría cuatro y cinco estrellas. Según Vargas (2015) la gestión ambiental en hoteles de Cancún, es una labor de participación directiva, de los empleados, autoridad gubernamental y clientes, lo que se asemeja con los conceptos mencionados por los actores claves para el caso de Puerto Vallarta: participación directiva, cliente y relación con la autoridad. En el análisis realizado por Wan et al. (2017) el motivador más mencionado es el de ahorro económico, coincidiendo con la visión de todos los actores clave hoteleros entrevistados en Puerto Vallarta.

\section{Conclusiones}

El objetivo de la investigación fue analizar las motivaciones y limitaciones para realizar gestión ambiental en los hoteles de Puerto Vallarta, de acuerdo con la visión de los actores clave involucrados, se concluye que el cliente se considera como un factor importante para llevar a cabo GAH, más no tiene aún el peso suficiente para que se realice la gestión por ese motivo exclusivamente.

La cooperación entre sectores es motivación externa para el personal hotelero cuando esta existe entre las partes; se vuelve una limitación externa en su ausencia, es decir, cuando entre los organismos gubernamentales y el sector hotelero no existen acuerdos. Por otro lado, debe existir una participación directiva para que se realicen buenas prácticas ambientales, independientemente de la categoría del hotel. La ausencia de esta característica anula las acciones para la protección ambiental.

El actor clave entrevistado proporciona la información según como la conoce. Al no haber presentado un listado estructurado para la elección de cuáles buenas prácticas ambientales realiza, el sujeto entrevistado proporciona únicamente información de la que es consciente, no de lo que el entrevistador piensa que es una respuesta correcta. Los actores clave identificados se relacionan directamente con la gestión ambiental en un hotel.

Existe cierta presión por parte del cliente, según los actores aquí entrevistados; para comprobar lo anterior se sugiere un posterior estudio enfocado en las preferencias del turista para la elección de hotel. La motivación interna para realizar GAH es más factible cuando el hotel forma parte de un corporativo que cuenta con estándares, y usualmente las cadenas hoteleras son quienes establecen un sistema de gestión ambiental más robusto y a largo plazo por los recursos económicos que poseen, sería interesante conocer si realmente una cadena hotelera puede volverse una empresa a imitar por sus acciones y conocimientos técnicos.

La falta de conocimiento técnico no es valorada como una limitación para el personal hotelero entrevistado, sin embargo, los consultores y organismos gubernamentales si lo visualizan como una limitación.

Los establecimientos implementan buenas prácticas ambientales cuando los directivos están convencidos en establecer acciones de protección ambiental; caso contrario, la falta de participación de los di- 
rectivos se vuelve una limitación para realizar gestión ambiental. La gestión ambiental hotelera existe como proceso planificado en una minoría de hoteles en Puerto Vallarta, no solo por la falta de inspección a los establecimientos de hospedaje, sino también por falta de interés por los directivos del establecimiento y por el costo de la implementación de las buenas prácticas ambientales y certificaciones.

Entre más recursos económicos posea el establecimiento, es más factible que se desarrolle gestión ambiental y se reconozcan sus beneficios económicos. El factor económico es una limitación cuando falta presupuesto para realizar inversiones y una motivación cuando la actividad genera ahorros económicos.

Si aumenta la inspección de la regulación ambiental en los hoteles y la posible aplicación de incentivos para realizarla, la GAH aumentará.

De acuerdo a la categoría del hotel se puede concluir que:

Para hoteles con categoría dos estrellas:

- La presión que el cliente ejerce para que se realice una práctica ambiental o se obtenga una certificación en esta materia, es prácticamente nula

- El ahorro económico representa una motivación interna en cuanto a la aplicación de buenas prácticas para minimizar costos, siempre y cuando los proyectos sean pequeños y moderados.

- Para el caso de los hoteles con categoría tres estrellas:

- El ahorro económico es una motivación interna, debe estar integrado y convenir en cualquier proyecto a implementar

- El cliente es una motivación externa.

- Desde el punto de vista de los hoteles cuatro y cinco estrellas:

- Mencionan como motivación interna los estándares del corporativo

- Realizan buenas prácticas ambientales o de obtención de certificaciones para cumplir con la legislación.

Finalmente, este trabajo presenta un caso de estudio en Puerto Vallarta, por lo tanto, los resultados pueden tener una generalización limitada. Sin embargo, su diseño permitió explorar en un destino turístico consolidado de sol y playa las limitantes y motivaciones de la gestión ambiental en hoteles, que permite comparar con otros casos en el mundo, además es el único trabajo que integra la visión de actores clave además del sector hotelero, al gobierno y consultores ambientales, lo que implica una contribución importante en el tema. En un futuro se sugiere ampliar y estratificar la muestra con el fin de hacer comparaciones robustas entre las diferentes categorías de hoteles.

\section{Referencias}

Almada, E. (2008). El análisis de actores. Metodología para el análisis contextual en Bibliotecología y Estudios de la Información. XXVI Coloquio de Investigación Bibliotecológica. UNAM. Ciudad de México. http://iibi.unam.mx/voutssasmt/documentos/xxvi_coloquio_cuib.pdf 
Álvarez, G., Burgos Jiménez, J. y Céspedes Lorente, J. (2001). An analysis of environmental management, organizational context and performance of Spanish hotels. The International Journal of Management Science, 29 (6), 457 - 471. https://doi.org/10.1016/S0305-0483(01)00033-0

Andrade, E. y Chávez-Dagostino, R. M. (2007). Entre el patrimonio natural y el cultural habita el mito: Isla del río Cuale (Puerto Vallarta, Jalisco, México). PASOS, Revista de Turismo y Patrimonio Cultural, 5(1), 111-124. https://doi.org/10.25145/j.pasos.2007.05.009

Andrés, J. M. y Torre, M. (2010). Las buenas prácticas ambientales en turismo. Anuario de Estudios en Turismo, Investigación y Extensión, 6 (10), 46-59. http://rdfatu.uncoma.edu.ar:8080/xmlui/handle/123456789/189

Aznar, J., Sayeras, J., Galiana, J. y Rocafort, A. (2016). Sustainability Commitment, New Competitors' Presence, and Hotel Performance: The Hotel Industry in Barcelona. Sustainability, 8(8), 1-13. https://doi.org/10.3390/su8080755

Bagur-Femenias, L., Celma, D. y Patau, J. (2016). The Adoption of Environmental Practices in Small Hotels. Voluntary or Mandatory? An Empirical Approach. Sustainability, 8(7), 1-14. https://doi. org/10.3390/su8070695

Baños Francia, A., Huízar-Sánchez, M. de los A., \& López, J. L. (2020). Políticas de gestión turística , transformación urbana y patrimonio en Puerto. PatryTer - Revista Latinoamericana e Caribenha de Geografia e Humanidades, 3(6), 51-65.

Bruns-Smith, A., Choy, V., Chong, H. y Verma, R. (2015). Environmental sustainability in the hospitality industry: Best practices, guest participation, and customer satisfaction. Cornell Hospitality Report, 15(3), 6-16. http://dx.doi.org/10.1108/IJCHM-02-2015-0076

Carasuk, R., Becken, S. y Hughey, K. F. D. (2013). Exploring values, drivers and barriers as antecedents of implementing responsible tourism. Journal of Hospitality \& Tourism Research, 40(1), 19-36. https:// doi.org/10.1177/1096348013491607

Céspedes, J. y De Burgos, J. (2004). Un análisis de las dimensiones de la gestión ambiental en los servicios hoteleros. Revista de dirección, organización y administración de empresas, 30, 5-15.

Chan, E. (2008). Barriers to EMS in the hotel industry. International Journal of Hospitality Management, 27(2), 187-196. https://doi.org/10.1016/j.ijhm.2007.07.011

Chan, E. S. W. y Hsu, C. (2016). Environmental management research in hospitality. International Journal of Contemporary Hospitality Management, 28(5), 886-923. http://dx.doi.org/10.1108/ IJCHM-02-2015-0076

Chan, E., Okumus, F. y Chan, W. (2018). Barriers to environmental technology adoption in hotels. Journal of Hospitality \& Tourism Research, 42(5), 829-852. https://doi.org/10.1177/1096348015614959

Chiappetta, C., Lopes de Sousa, A., Govindan, K., Pignatti de Freitas, T., Devika Kannan, F. y Latan, H. (2016). Barriers to the adoption of green operational practices at Brazilian companies: effects on green and operational performance. International Journal of Production Research, 54(10), 3042 3058. https://doi.org/10.1080/00207543.2016.1154997

Cingoski, V. y Petrevska, B. (2018). Making hotels more energy efficient: the managerial perception. Economic Research-Ekonomska Istraživanja, 31(1), 87-101. https://www.researchgate.net/deref/ https\%3A\%2F\%2Fdoi.org\%2F10.1080\%2F1331677X.2017.1421994 
Cramer, J. (1998). Environmental management: from "fit" to "stretch". Business Strategy and the Environment. 7(3), 162-172. https://doi.org/10.1002/(SICI)1099-0836(199807)7:3<162::AIDBSE149>3.0.CO;2-Q

Díaz-Bravo, L., Torruco-García, U., Martínez-Hernández, M. y Varela-Ruíz, M. (2013). La entrevista, recurso flexible y dinámico. Investigación en Educación Médica, 2(7), 162-167. http://riem.facmed. unam.mx/sites/all/archivos/V2Num03/09_MI_LA\%20_ENTREVISTA.pdf

Dunk, R., Gillespie, S. y MacLeod, D. (2016). Participation and retention in a green tourism certification scheme. Journal of Sustainable Tourism, 24(12), 1585-1603. https://doi.org/10.1080/09669 582.2015 .1134558

Emre, Y., Ergün, E. y Selami, M. (2015). How business environment hotels compete in Turkey? An evaluation from managers 'perspectives'. European Journal of Tourism, Hospitality and Recreation, 6(2), 117-138. https://pingpdf.com/download/how-business-environment-hotels-compete-inturkey_5a386886d64ab2d826c5a413.html

Ervin, D., Wu, J. Khanna, M., Jones, C. y Wirkkala, T. (2012). Motivations and Barriers to Corporate Environmental Management. Business Strategy and the Environment, 22(6), 390-409. https://doi. org/10.1002/bse.1752

Fleig, A. N., Silveira, V., Cruz, A., Stoll, B. y Kirchner, R.M. (2015). Percepção da gestão ambiental no setor hoteleiro do município de São Gabriel, RS. Revista Electrónica en Gestión, educación y tecnología ambiental, 19 (3) 659-666. https://periodicos.ufsm.br/reget/article/viewFile/17818/pdf

Higgins-Desbiolles, F. (2018). Sustainable tourism: Sustaining tourism or something more? Tourism Management Perspectives, 25, 157 - 160. https://doi.org/10.1016/j.tmp.2017.11.017

Hutchins, J., Sinha, M. y Nandan, S. (2019). The sustainability route to corporate legitimacy. Journal of Global Scholars of Marketing Science, 29(1), 15-24. https://doi.org/10.1080/21639159.2018.1551727

Kirk, D. (1995). Environmental management in hotels. International Journal of Contemporary Hospitality Management, 7(6), 3-8. https://doi.org/10.1108/09596119510095325

López, L. y Vargas, E. (2013). Evaluación preliminar de la gestión ambiental en hoteles del destino turístico de Varadero, Cuba. Teoría y Praxis, (13), 133-150. https://doi.org/10.22403/UQROOMX/TYP13/06

Mihalič, T. (2000). Environmental management of a tourist destination a factor of tourism competitiveness. Tourism Management, 21(1), 65-78. https://doi.org/10.1016/S0261-5177(99)00096-5

MinCIT. (2014). Guía de buenas prácticas para prestadores de servicios en turismo de naturaleza. Programa de Transformación Productiva del Ministerio de Comercio, Industria y Turismo. https://www. ptp.com.co/CMSPages/GetFile.aspx?guid=f01cde94-55d0-4d6d-b397-1cdc4d682696

Navarrete, I. y Zanfardini, M. (2014). Los instrumentos de gestión ambiental de carácter voluntario, como estrategia de marketing responsable en hotelería. VI Congreso Latinoamericano de Investigación Turística. Patagonia, Argentina. http://revele.uncoma.edu.ar/htdoc/revele/index.php/condet/article/view/2376/59026

Organización de las Naciones Unidas. (2000). Resolución aprobada por la Asamblea General (A/55/L.2). Asamblea General de las Naciones Unidas. https://www.un.org/spanish/milenio/ares552.pdf

Organización de las Naciones Unidas. (2019). Objetivos y metas de desarrollo sostenible, 17 objetivos para transformar nuestro mundo. Página oficial de las Naciones Unidas. https://www.un.org/sustainabledevelopment/es/sustainable-development-goals 
Ortiz, Y., Vargas, E., Nava, R. y Castillo, M. (2016). Los stakeholders de la industria hotelera: una clasificación a partir de sus intereses ambientales. Universidad y Empresa, 18(30), 97-120. http://doi. org/10.12804/rev.univ.empresa.30.2016.05

Otzen, T., y Manterola, C. (2017). Técnicas de Muestreo sobre una Población a Estudio. International Journal of Morphology, 35(1), 227-232. https://doi.org/10.4067/S071795022017000100037

Peiró-Signes, A., Segarra-Oña, M., Verma, R.., Mondéjar-Jiménez, J. y Vargas-Vargas, M. (2014). The Impact of Environmental Certification on Hotel Guest Ratings. Cornell Hospitality Quarterly, 55(1), 40-51. https://doi.org/10.1177\%2F1938965513503488

Pérez, J. (2010). La política ambiental en México: Gestión e instrumentos económicos. El Cotidiano, (162), 91-97.

Prats, L. (2011). La viabilidad turística del patrimonio. PASOS, Revista de Turismo y Patrimonio Cultural, 9(2), 249-264. https://doi.org/10.25145/j.pasos.2011.09.023

Reid, S., Johnston, N. y Patiar, A. (2017). Coastal resorts setting the pace: An evaluation of sustainable hotel practices. Journal of Hospitality and Tourism Management, 33, 11-22. https://doi.org/10.1016/j. jhtm.2017.07.001

Rodríguez-Becerra, M. y Espinoza, G. (2002). Gestión ambiental en América Latina y el Caribe. Evolución, tendencias y principales prácticas. Banco Interamericano de Desarrollo.

Rubin, S., White, D., Lee, W. y Basile, G. (2012). Antecedents of Effective Environmental Management in a Hotel Setting: A Test of the Value-Belief-Norm Theory. 2012 Travel and Tourism Research Association: TTRA International Conference (junio 17-19, pp. 1-7). TTRA, Virginia. https://scholarworks. umass.edu/ttra/2012/Visual/6/

Sánchez-Medina, P. S., Díaz-Pichardo, R. y Cruz-Bautista, M. (2015). Stakeholder influence on the implementation of Environmental Management practices in the hotel industry. International Journal of Tourism Research, 18(4), 387-398. https://doi.org/10.1002/jtr.2056

Scartascini, S. G y Rodríguez, L.A. (2011). Puerto Vallarta en su historia y cultura. En Sociedad, naturaleza y turismo ( $1^{\mathrm{a}}$ ed., pp. 147-150). Guadalajara: Universidad de Guadalajara.

Secretaría de Turismo Jalisco. (2019). Estadísticas del sector turístico | Secretaría de Turismo. Afluencia. Anuario 2019. https://secturjal.jalisco.gob.mx/invierte-en-jalisco/estadisticas

Secretaría de Turismo. (2016). Anuario Estadístico del Estado de Jalisco. Dirección General de Planeación y Desarrollo de Destinos Turísticos, Jalisco. https://secturjal.jalisco.gob.mx/invierte-en-jalisco/estadisticas

Toledo, A., y Gopar, N. (2012). Stakeholders y prácticas ambientales en las PyMEs hoteleras. XVI Congreso Internacional de Investigación en Ciencias Administrativas: Retos de las ciencias administrativas desde las economías emergentes: Evolución de sociedades (22 al 25 de mayo, pp. 1-20). Academia de Ciencias Administrativas A.C. Estado de México. http://acacia.org.mx/busqueda/pdf/03_PF344_ Pr_cticas_Ambientales.pdf

Valenzuela, M. (2017). La sostenibilidad ambiental del sector hotelero español. Una contribución al turismo sostenible entre el interés empresarial y el compromiso ambiental. ARBOR Ciencia, pensamiento y cultura, 193(785), Article a403. http://dx.doi.org/10.3989/arbor.2017.785n3009

Vargas, E. E. (2015). Responsabilidad social empresarial y gestión ambiental en el sector hotelero. Universidad Autónoma del Estado de México. 
- Revista de Ciencias Sociales y Humanidades. ISSN-P: 0188-9834 ISSN-E:2395-8669.

Vargas, E. E. y Olivares, A. (2012). Responsabilidad ambiental empresarial: el caso de Hotelera Posadas. El Periplo Sustentable, (22), 163-188.

Vargas, E., Zizumbo, L.; Carlos, F. y Serrano, R. (2011). Gestión ambiental en el sector turístico mexicano: Efectos de la regulación en el desempeño hotelero. Cuadernos de Administración, 24 (42), 183-204. https://www.redalyc.org/articulo.oa?id=205/20520042009

Walker, H., Di Sisto, L. y McBain, D. (2008). Drivers and barriers to environmental supply chain management practices: Lessons from the public and private sectors. Journal of Purchasing \& Supply Management, 14 (1), 69-85. https://doi.org/10.1016/j.pursup.2008.01.007

Wan, Y. K.P., Chan, S. H. J. y Huang, H. L.W. (2017). Environmental awareness, initiatives and performance in the hotel industry of Macau. Tourism Review, 72 (1), 87-103. https://doi.org/10.1108/ TR-06-2016-0016 